\title{
Sport Science Is a Team Effort
}

In the world of sport, we frequently hear and talk about individual and team sports; often they are even considered as being opposed to each other-individual sports versus team sports. In the same manner, sport scientists are also allocated to one of 2 possible categories, for example, an individual-sport physiologist/biomechanist/nutritionist or a team-sport performance analyst. Nevertheless, the fundamental set of movement and athletic skills required for elite sport performance can be applied to excel in an individual sport or put at the service of a team. Professional road cyclists racing in a Grand Tour, for instance, are ranked every day of competition in an individual stage classification as well as a general classification. However, most of the participants perform the role of "domestiques," riding for the benefit of their team and team leader at the expense of their own personal glory. In some team sports like basketball and soccer, the final score is the main outcome of a match, but individual players are then put in the spotlight as their personal statistics are displayed: points or goals scored, field goal percentages, total distance covered, rebounds, personal fouls, and so forth. Nevertheless, everyone realizes that the "star players" would be ineffective without the support of their teammates.

The same applies to sport scientists in general and sport physiologists in particular. Our set of professional skills can be put at the service of individual athletes, sport teams, or both. They can also contribute to the success of an academic department or research institution. When we apply for an academic position, a research grant, or a job as a practitioner for a professional team or sport organization, our merits are assessed as individuals, but as a general rule, whether we work in academia or in professional or Olympic sports, we always need to "perform" as team players.

My 2017 editorial titled "Winning the BIG medals"" listed the key qualities of sport scientists that make a significant contribution to medal-winning performances, according to medal-winning coaches. Some of these fundamental qualities were directly related with the idea of sport scientists being part of a team effort: ability to be part of a group, not the "star" of the group; ability to relate to coach and athlete; availability; helping a project, not changing it; and readiness for cooperation. These qualities are, of course, not innate and need to be trained and developed over time. Moreover, they also apply to sport scientists working in an academic setting and/or research institution. Just consider the following numbers accumulated over my 30-year career as a researcher in sport science:

- 213: the number of co-authors in my published scientific articles

- 23: the countries of origin of these co-authors (international collaborations are now easier than ever)

- 2545: the number of study participants (meta-analyses not included; there is nothing we could do in human research without the voluntary contribution of study participants!)
- 94: the number of colleagues and collaborators named in the acknowledgments of my published articles

- 78: the number of institutions that have provided financial support for my studies in one way or another (these include universities, private companies, clinics and laboratories, sport ministries, ministries of education and research, high-performance centers, research foundations, professional and amateur sport clubs, sport federations, and Olympic committees)

- 4161: the number of references used in my published articles (like Sir Isaac Newton, we all see further by standing on the shoulders of giants)

- 300: the approximate number of peers who have volunteered their time and expertise over the years to review my scientific articles before they were published

This level of activity and output has only been possible by the collaborative nature of sport science both in the field with coaches and athletes and in academic circles. These metrics are easily generated, but the challenge is to identify and articulate the impact of sport science. For instance, these numbers were achieved while simultaneously helping individual athletes and sport teams achieve their performance targets at international and Olympic levels either as a coach or as a sport physiologist. High-level sporting programs and teams expect and demand sport-science support that makes a difference. Similarly, universities and other research institutions are looking for funding, reputation, media and social media exposure, and demonstrated impact at a community and organizational level. There is a lot more at stake than simply delivering a service.

Career progression and success are driven by a concerted effort for professional advancement and opportunity. My advice to aspiring and established sport scientists as individuals is to direct their efforts toward improving their own sets of fundamental skills to become better professionals (ie, better sport practitioners, academics, researchers) but to always be generous and put their know-how and expertise at the service of their teams and the wider international sport-science community. Submitting work for peerreviewed publication, contributing to professional and academic conferences and meetings, volunteering on journal editorial boards, and/or reviewing the work submitted by their peers will benefit individual sport scientists and the broader sport-science community.

Iñigo Mujika, IJSPP Associate Editor, University of the Basque Country (UPV/EHU)

\section{Reference}

1. Mujika I. Winning the BIG medals. Int J Sports Physiol Perform. 2017;12(3):273-274. PubMed ID: 28504590 doi:10.1123/ijspp. 2017-0016 